\title{
Uso do Atlas Esgotos: Despoluição de Bacias Hidrográficas no desenvolvimento de trabalhos científicos
}

\author{
Maíra Araújo de Mendonça Lima \\ Engenheira Ambiental e Sanitarista - Universidade do Estado do Rio de Janeiro \\ $\triangle$ mairalima.90@gmail.com \\ Bruna Magalhães de Araujo \\ Engenheira Civil - Universidade do Estado do Rio de Janeiro - PEAMB/UERJ \\ Sérgio Rodrigues Ayrimoraes Soares \\ Engenheiro Civil - Superintendente de Planejamento de \\ Recursos Hídricos da Agência Nacional de Águas - ANA \\ Ana Silvia Pereira Santos \\ Engenheira Civil - D.Sc. Professora do Departamento de Engenharia Sanitária e do \\ Meio Ambiente da Universidade do Estado do Rio de Janeiro - DESMA/UERJ
}

Recebido em 4 de março de 2020

Aceito em 2 de julho de 2020

\begin{abstract}
Resumo: para desenvolvimento de suas respectivas pesquisas. revisão sistemática.

\section{Use of the Sewer Atlas: Depollution of Water Basins in the development of scientific works}

O documento "Atlas Esgotos: Despoluição de Bacias Hidrográficas" foi publicado pela Agência Nacional de Águas (ANA), no ano de 2017 e apresenta um caráter de grande relevância para o desenvolvimento da gestão de recursos hídricos e saneamento ambiental no Brasil. Sua abordagem é abrangente e apresenta dados sanitários de todos as 5.570 sedes urbanas brasileiras. Dessa forma, o presente trabalho teve como objetivo, avaliar a inserção dos dados apresentados por este documento, no desenvolvimento de trabalhos científicos. Para tanto foi utilizada como metodologia a Revisão Sistemática da Literatura, por meio de buscas realizadas através da palavra chave "Atlas Esgotos" e suas variações. Deste modo, para realização da pesquisa, foram analisadas publicações efetuadas no período entre 2017 e 2019, utilizando o Google Scholar como principal mecanismo de busca. De acordo com o estudo realizado, foram encontradas e selecionadas 129 publicações, onde $12 \%$ são provenientes de periódicos, $5 \%$ de livros, $33 \%$ de anais de congressos, e $50 \%$ de trabalhos de conclusão de curso. No que diz respeito à forma como o Atlas Esgotos tem sido utilizado nas pesquisas científicas, concluiuse que $71 \%$ das publicações realizam apenas citação dos seus dados, $25 \%$ utilizam os dados a título de comparação com outras fontes ou demais cenários, e $4 \%$ aplicam, de fato, os dados do Atlas Esgotos

Palavras-chave: Saneamento ambiental, recursos hídricos, instrumento estratégico de gestão,

\begin{abstract}
:
The document Atlas Esgotos: Despoluição de Bacias Hidrográficas (Sewers Atlas: Depollution of Water Basins) was published by Water National Agency (ANA), at 2017 and presents great relevance for the development of water resources and sanitation management in Brazil. Its approach is comprehensive and presents sanitary data from all 5,570 Brazilian municipalities. Thus, the research aims evaluate
\end{abstract}


the insertion of the data presented in this document, in the development of scientific works. Thus, the methodology called Systematic Literature Review was used through searches carried out using the keyword Atlas Esgotos and its variations. To conduct the research, scientific documents published in the period between 2017 and 2019 were analyzed, using Google Scholar as the main search engine. According to the study, 129 documents were found and selected, where $12 \%$ are from journals, $33 \%$ from conference annals, and $50 \%$ from course completion papers. With regard to evaluation the way that the Sewer Atlas has been used in scientific research, it was concluded that $71 \%$ of the publications only quote the data, $25 \%$ used it for comparison with other sources or other scenarios and $4 \%$ applied, in fact, the data from the Sewer Atlas for the development of their respective research.

Keywords: Sanitation, water resources, strategic management tool, systematic review.

\section{Uso del Atlas de Alcantarillado: Despliegue de Cuencas Hidrográficas en el desarrollo de trabajos científicos}

\section{Resumen:}

El documento Atlas Esgotos: Despoluição de Bacias Hidroráficas (Atlas del Alcantarillado: Despliegue de Cuencas Hidrográficas) fue publicado por la Agencia Nacional del Agua (ANA) en 2017 y tiene un carácter de gran relevancia para el desarrollo de la gestión de los recursos hídricos y el saneamiento ambiental en Brasil. Su enfoque es integral y presenta datos sanitarios de los 5.570 centros urbanos brasileños. Por lo tanto, este estudio tuvo como objetivo evaluar la inserción de los datos presentados por este documento, en el desarrollo de trabajos científicos. Para ello, se utilizó la Revisión sistemática de la literatura como metodología, mediante búsquedas realizadas con la palabra clave "Atlas Esgotos" y sus variaciones. Por lo tanto, para realizar la investigación, se analizaron las publicaciones realizadas en el período comprendido entre 2017 y 2019, utilizando Google Scholar como el principal motor de búsqueda. Según el estudio, se encontraron y seleccionaron 129 publicaciones, de las cuales $12 \%$ provienen de revistas cientificas, $5 \%$ de libros, $33 \%$ de actas de congresos y $50 \%$ de trabajos de finalización de cursos. Con respecto a cómo se ha utilizado Atlas del Alacantarillado en la investigación científica, se concluyó que el $71 \%$ de las publicaciones solo citan sus datos, $25 \%$ usa los datos como una comparación con otras fuentes u otros escenarios, y $4 \%$ aplica, de hecho, los datos del Atlas Esgotos para el desarrollo de sus respectivas investigaciones.

Palabras clave: Saneamiento ambiental, recursos hídricos, herramienta de gestión estratégica, revisión sistemática.

\section{INTRODUÇÃO}

O Saneamento Ambiental, de maneira geral, trata-se de um conjunto de ações de infraestrutura, tais como Sistema de Abastecimento de Água, Sistema de Esgotamento Sanitário, Gestão de Resíduos Sólidos e Gestão de Águas Pluviais, para fins de proteção ambiental e de promoção à saúde da população. Dentre os princípios fundamentais relacionados à prestação dos serviços públicos de saneamento básico destacam-se os seguintes: universalização dos sistemas, segundo uma ampliação progressiva do acesso de todos os domicílios ocupados; articulação com as políticas de desenvolvimento urbano e regional, de habitação, de combate à pobreza, de proteção ambiental e de promoção à saúde; e eficiência e sustentabilidade econômica (BRASIL, 2007). 
De forma a alcançar a universalização, articulando políticas de desenvolvimento socioeconômico, de maneira sustentável e integrada, é de extrema relevância o desenvolvimento de documentos públicos que retratem com fidelidade, o cenário da situação do saneamento ambiental no país e em suas diferentes regiões geográficas e/ou hidrográficas. Esses instrumentos organizam dados de forma a transformá-los em informações necessárias e absolutamente fundamentais ao planejamento e às tomadas de decisão. 0 planejamento das ações potencializa a gestão dos serviços públicos, propicia melhorias para a população, corrige problemas imediatos, direciona ações de médio e longo prazo e reduz a necessidade de ações emergenciais (YÉVENES-SUBIATRE, 2010; LISBOA et al., 2013; HELLER e NASCIMENTO, 2005).

No caso desses instrumentos no Brasil, em relação ao esgotamento sanitário, destacam-se o Sistema Nacional de Informações sobre Saneamento (SNIS) desenvolvido anualmente pela Secretaria Nacional de Saneamento Ambiental do Ministério do Desenvolvimento Regional (SNS/MDR) (BRASIL, 2019) e o Atlas Esgotos: Despoluição de Bacias Hidrográficas desenvolvido pela Agência Nacional de Águas (ANA, 2017).

Para a elaboração anual do Diagnóstico dos Serviços de Água e Esgotos, os dados dos serviços são fornecidos ao SNIS pelas prestadoras de serviços. Segundo Costa (2015), os dados do SNIS demonstram certa fragilidade, pois se tratam de abordagens auto declaratórias dos prestadores de serviços, que seguem recomendações apresentadas em um manual, de forma a auxiliar a geração de informações com maior grau de confiança e exatidão.

O Atlas Esgotos: Despoluição de Bacias Hidrográficas foi publicado em 2017, pela ANA, com dados referentes aos sistemas de esgotamento sanitário no território nacional, baseado nos seguintes princípios: i) caracterização da situação do esgotamento sanitários dos municípios; ii) ampliação do conhecimento sobre o tratamento de esgotos para redução dos impactos na saúde pública; iii) criação de soluções complementares com vistas à melhoria sustentável da qualidade dos corpos hídricos; iv) qualificação da gestão operacional das soluções de esgotamento sanitário para viabilização de alternativas e investimento; v) fortalecimento do processo de planejamento e gestão de recursos hídricos e saneamento.

A elaboração do respectivo documento contou com um levantamento de dados coletados entre os anos de 2013 e 2015, nos 5.570 municípios brasileiros, separados em dois grupos. O Grupo 1, que contou com levantamento de dados primários por meio de visitas de 
campo e reuniões técnicas, reuniu os municípios com prestadores de serviço institucionalizados, a partir de companhias estaduais, autarquias municipais e concessionárias privadas. Neste grupo, ainda foram incluídos os municípios sem prestador de serviço institucionalizado, mas com população urbana acima de 50.000 habitante. Já o Grupo 2 abordou os municípios com população inferior a 50.000 habitantes e utilizou dados secundários.

Quando comparado com outros estudos fundamentados apenas em dados secundários, o Atlas esgoto apresenta um diferencial, visto que para sua elaboração realiza um refinamento de informações, juntamente com o levantamento de dados primários, relativo ao grupo que concentra a maior parcela da população. Neste cenário, o Atlas tornase um documento de referência a ser utilizado para a tomada de decisão na gestão de recursos hídricos e saneamento, na orientação dos investimentos no tratamento do esgoto urbano e no planejamento estratégico para a implantação de novos sistemas e ampliação de sistemas existentes, com vistas à universalização. Diante da crescente incorporação de estudos técnicos-científicos no planejamento das ações de saneamento, o presente artigo teve como objetivo avaliar a inserção dos dados apresentados pelo Atlas Esgotos no desenvolvimento de trabalhos científicos.

\section{MATERIAL E MÉTODOS}

Para o desenvolvimento do trabalho, em duas etapas, foi utilizada a metodologia conhecida por Revisão Sistemática da Literatura. Segundo Cook et al. (1997) essa metodologia é baseada na aplicação de métodos com alto rigor científico, podendo alcançar melhores resultados e reduzir erros, além de permitir ao pesquisador compilar dados, refinar hipóteses, estimar tamanho de amostras, definir melhor o método de pesquisa a ser adotado, e por fim definir direções para futuras pesquisas. 


\section{definidas \\ Etapa 1 - Pesquisa nos bancos de dados selecionados a partir das palavras chave}

De acordo com a metodologia de revisão sistemática proposta por Sampaio e Mancini (2007), no presente trabalho foram realizadas buscas de publicações eletrônicas técnicocientíficas, em um recorte temporal pré-estabelecido, com palavras chaves específicas que pudessem remeter à utilização do Atlas Esgotos como ferramenta de estudo, em bases de dados selecionadas.

O recorte temporal foi definido para os anos de 2017 a 2019, em função da data de publicação do documento objeto do estudo (Atlas Esgotos), em 2017, até a finalização da redação do presente artigo (setembro de 2019). Inicialmente, foi empregada na busca, somente a palavra chave "Atlas Esgotos". Porém, ao observar que diversos autores adotaram nomenclaturas diferentes para o mesmo documento, foi realizada buscas com variações da palavra chave. Por fim, as bases acadêmicas de pesquisa foram adotadas principalmente em função da facilidade de acesso e interação com o usuário, da facilidade da busca por estudos publicados no idioma português, da qualidade para o refinamento de buscas, da confiabilidade e da cobertura.

A ferramenta de busca Google Scholar é bastante apropriada para pesquisas bibliográficas nas áreas das Ciências Exatas, da Terra e Engenharias, se apresentando dentre outras Mecanismos de Buscas Acadêmicas (MBAs), como sendo a melhor nos quesitos de cobertura e acessibilidade (BROPHY e BAWDEN, 2005; MEIER e CONKLING, 2008; BUCHINGER et al., 2014). Meho e Yang (2007) analisaram, além do Google Scholar, Web of Knowledge e Scopus, chegando à conclusão que a ferramenta Google foi a que retornou a maior quantidade de documentos únicos, ou seja, que não foram encontrados nos outros MBAs. Segundo Battistella et al. (2014), o Google Scholar apresenta tanto artigos publicados em revistas reconhecidas cientificamente, como também possibilita encontrar documentos publicados oriundos de outras fontes, como por exemplo, bibliotecas digitais ou repositórios digitais, ampliando a busca em diversos meios e domínios de publicação online.

Assim, o Google Scholar foi o principal mecanismo de busca adotado na presente pesquisa. Ainda, pela familiaridade com a ferramenta e o acesso concedido às Universidades, dos principais mecanismos pagos, o Portal Periódicos da CAPES (Coordenação de Aperfeiçoamento de Pessoal de Nível Superior) também foi adotado para complementação 
dos resultados. Por fim, os anais dos principais eventos científicos de Engenharia Sanitária ocorridos no Brasil, no recorte temporal adotado para a pesquisa e que são do conhecimento dos autores também foram utilizados.

De maneira resumida, a palavra chave "Atlas Esgotos" foi utilizada com as seguintes variações: Atlas de Esgoto, Atlas de Esgotos, Atlas do Esgoto, Atlas do Esgotos, Atlas Esgoto, Atlas Esgotos. E os bancos de dados adotados foram: Google Scholar, Portal de Periódicos da CAPES, Anais do XIV Simpósio Ítalo Brasileiro de Engenharia Sanitária e Ambiental (SIBESA), realizado em Foz do Iguaçu - Paraná em 2018 e Anais do 30 Congresso Brasileiro de Engenharia Sanitária (CBESA), realizado em Natal - Rio Grande do Norte em 2019. Ressalta-se que $29^{\circ} \mathrm{CBESA}$ não foi incorporado à pesquisa por ter ocorrido em data muito próxima à do lançamento oficial do Atlas Esgotos.

\section{Atlas}

\section{Etapa 2 - Análise dos documentos e suas diferentes abordagens em relação ao uso do}

Nesta Etapa foi realizada uma análise dos documentos selecionados na Etapa 01, em função da premissa de haver nestes, alguma referência ao objeto de estudo (Atlas Esgotos), de acordo com as diferentes palavras chave já mencionadas no item anterior. Como critério de exclusão, adotaram-se os seguintes: i) documentos que apareceram mais de uma vez nos diferentes bancos de dados; e ii) documentos que citavam o Atlas Esgotos somente nas referências, sem fazer qualquer menção aos seus dados ao longo do texto. Ainda, dois artigos foram incluídos manualmente por serem conhecidos pelos autores e não terem aparecido nas buscas realizadas nas bases de dados adotadas na pesquisa. Isso ocorreu possivelmente por serem ainda publicações muito recentes e que não foram incorporadas aos bancos de dados consultados.

Após a seleção das publicações os documentos foram classificados de acordo com os seguintes itens, em planilha eletrônica:

- $\quad$ Banco de dados: Periódicos da CAPES; Google Acadêmico; XIV SIBESA; ou 30ํㅡㄴ Congresso da ABES; 
- Palavra-chave: Atlas de Esgoto, Atlas de Esgotos, Atlas do Esgoto, Atlas do Esgotos, Atlas Esgoto ou Atlas Esgotos;

- $\quad$ Dados do estudo: Título, autor, ano de publicação e local do estudo;

- Tipo de publicação: artigo de congresso, artigo de revista, dissertação de mestrado, tese de doutorado, documento público, monografia de especialização Latu Sensu, trabalho de conclusão de curso de graduação, trabalho de disciplina e livro (capítulo);

- $\quad$ Tema da pesquisa: Saneamento ou outros; e

- Forma de abordagem do documento base do estudo (Atlas Esgotos): somente citação, adoção dos dados para comparação com outras fontes ou demais cenários, ou uso dos dados para desenvolvimento do trabalho.

Diante do exposto nas duas etapas da metodologia foi possível realizar uma discussão em relação à distribuição dos resultados obtidos, de acordo com a classificação descrita anteriormente. Essa discussão levou ao entendimento das diferentes formas como o Atlas Esgotos tem sido usado nos trabalhos técnicos científicos e seu potencial para de fato, ser adotado como instrumento de planejamento para tomada de decisão.

\section{RESULTADOS} definidas

Etapa 1 - Pesquisa nos bancos de dados selecionados a partir das palavras chave

Em relação às publicações encontradas na presente pesquisa, na Figura 1, apresentase o fluxograma referente a seleção dos respectivos documentos analisados. 
Figura 1 - Fluxograma de seleção dos documentos referentes à revisão sistemática no período de janeiro de 2017 a setembro de 2019.

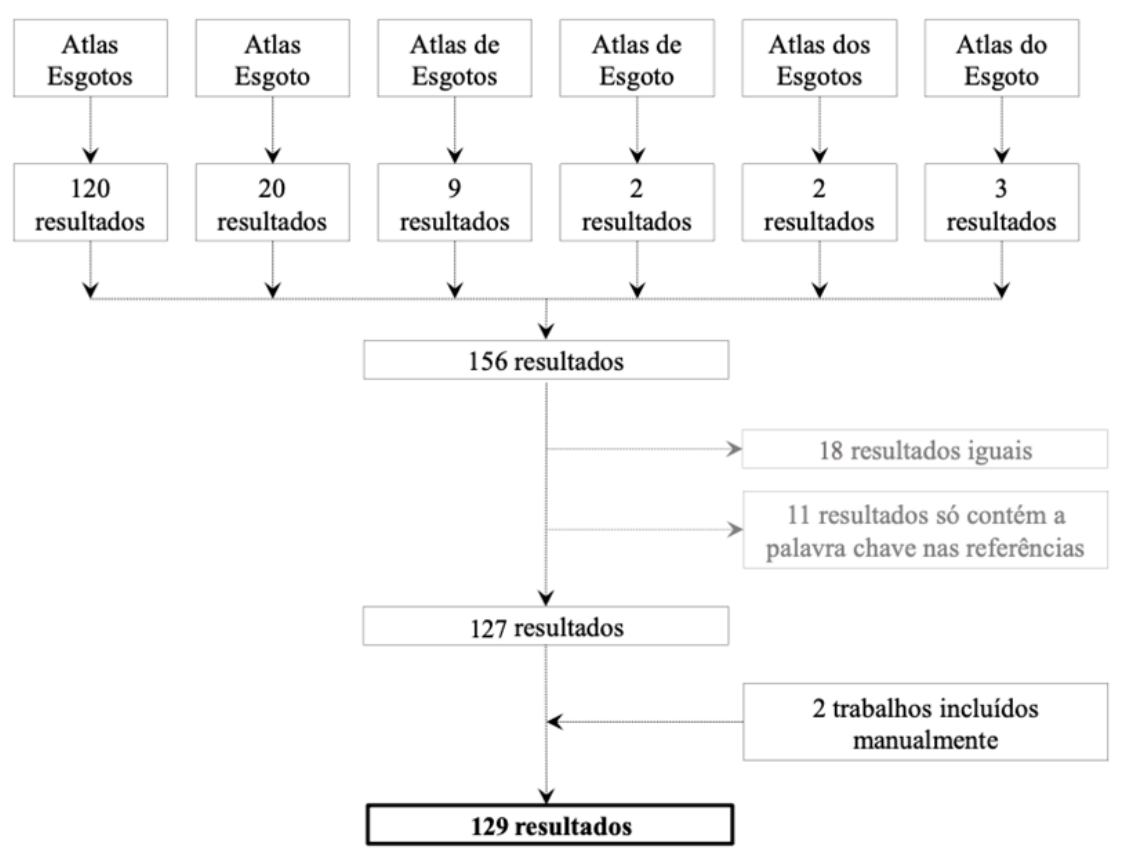

Fonte: Autores, 2019.

\section{Etapa 2 - Análise dos documentos encontrados e suas diferentes abordagens em relação ao uso do Atlas}

Para esta etapa, foram avaliados os documentos obtidos a partir dos critérios estabelecidos e organizados de acordo com a classificação descrita anteriormente na metodologia. Inicialmente, todos os trabalhos analisados no presente estudo foram categorizados de acordo com os objetivos definidos:

- Objetivo 1 - Dados somente citados:

Alencar e Zaneti (2018); Amaral e Tavares (2018); do Amaral et al. (2018); do Amaral et al. (2019); Armani et al. (2018); Ávila (2018); Barra (2018); Bonetti (2018); Borba e Franco (2018); Bringer et al. (2018); Brito (2018); Brito et al. (2019); Bucco (2018); Bucco et al. (2018); Cabral (2018); Callisto et al. (2019); Camara et al. (2019); Caminada (2019); Carli (2019); de Souza Carrasco (2018); Carvalho Júnior (2018); Cavalcanti et al. (2019); Chernicharo et al. (2018); Corrêa et al. (2019); Cruz (2018); Dantas et al. (2019); Dias NA (2018); Dias IC (2018); Dias et al. (2018); Duarte (2019); Esteves (2017); Faria et al. (2019); Ferreira (2019); Figueiredo (2019); Freire (2019); Garcia (2018); Miranda Gonçalves e Domingos (2019); Guinzani et al. (2018); Issa 
(2019); Lannes et al. (2018); Leal (2019); Lima (2018); Lima et al. (2019); Machado (2019); Maia (2018); Malzac e Silva (2019); Medeiros (2019); Medeiros et al. (2019); Melchior et al. (2018); Nascimento e Gonçalves (2019); Neves e Mol (2019a); Neves e Mol (2019b); Neves et al. (2018); Obraczka et al. (2019); Oliveira BVS (2018); Oliveira JC (2018); Oliveira et al. (2019); Parizotto (2018); Peixoto et al. (2018); Pereira FC et al. (2019); Pereira AC et al. (2019); Pertussatti (2018); Pigozzo (2018); Pizutti (2018); Pontes Filho (2018); Ramos et al. (2018); Reis et al. (2019); Ribeiro et al. (2018); Rosa Júnior et al. (2018); Rosário e Fernandes (2018); Santos (2017); Santos AA et al. (2019); Santos FNB et al. (2019); Silva APV (2018); Silva NS (2018); Silva RMQ (2018); Silva (2019); Silva et al. (2019); Silva Júnior e Trindade (2018); Silveira SF (2018); Silveira ACB (2018); Siqueira Júnior (2018); Soares (2019); Souza (2018); Souza (2019); Souza et al. (2019); Maistro Spolidorio (2018); Teixeira (2018); Viana (2018); Vieira (2018); Volpato et al. (2019); e Zanetoni Filho (2019).

- Objetivo 2 - Dados utilizados para efeito de comparação:

Aguiar et al. (2018); Almeida (2018); Almeida (2019); Ambrozevicius (2018); Araujo et al. (2018a); Araujo et al. (2018b); Araújo et al. (2019); Bursztejn (2018); Cerqueira (2019); Corrêa (2018); Do Amaral (2018); Franci (2018); Kato et al. (2019); Lima IM (2019); Lima MAM (2019); Lopes (2018); Monteiro et al. (2019); Moretto (2018); Motta Veiga (2019); Palasio (2019); Pessoa et al. (2018); Pessoa et al. (2019); Prestes (2018); Santos et al. (2018a); Santos et al. (2018b); Santos ASP et al. (2019); Silva JS (2018); Silva e Leite (2019); Silva Júnior et al. (2019a); Silva Júnior et al. (2019b); Teubner Junior et al. (2018); e Viana et al. (2019).

- Objetivo 3 - Dados adotados efetivamente no desenvolvimento da pesquisa:

Soares et al. (2017); Dutra e Smiderle (2018); Sales (2018); Dutra et al. (2018); e Paulo et al. (2019).

Posteriormente, analisaram-se a distribuição da quantidade de documentos publicados por ano, dentre o recorte temporal estabelecido, a distribuição da quantidade de documentos por tipo de publicação e a distribuição da quantidade de documentos por base de dados consultada.

No Gráfico 1, apresenta-se a distribuição da quantidade de documentos, de acordo com os objetivos de cada estudo e no Gráfico 2 apresenta-se a distribuição da quantidade de documentos em função do ano de publicação, no recorte temporal estabelecido. 
Gráfico 1 - Distribuição da quantidade de documentos pelo objetivo de estudo

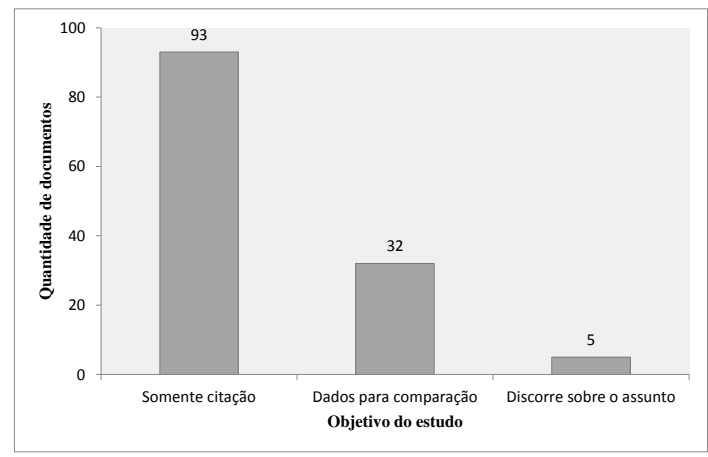

Fonte: Autores, 2019.
Gráfico 2 - Distribuição da quantidade de documentos por ano do recorte temporal estabelecido

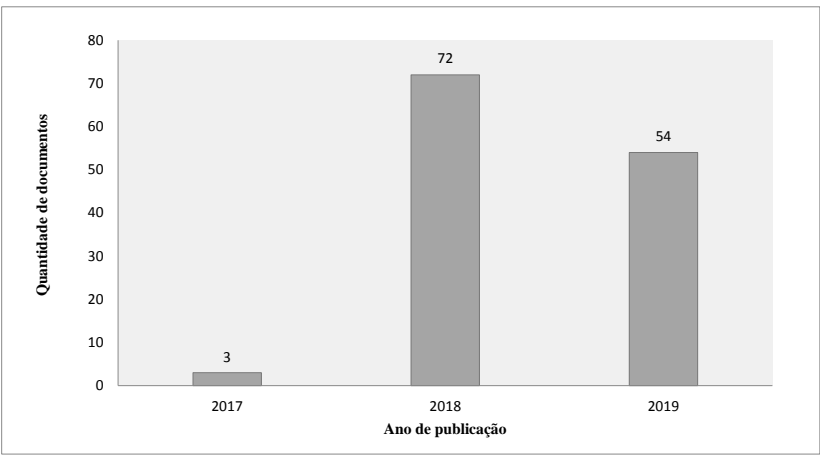

Fonte: Autores, 2019.

No Gráfico 3 está apresentada a distribuição referente a quantidade de documentos por tipo de publicação e no Gráfico 4, observa-se a distribuição da quantidade de documentos de acordo com a base de dados consultada.

Gráfico 3 - Distribuição da quantidade de documentos por tipo de publicação

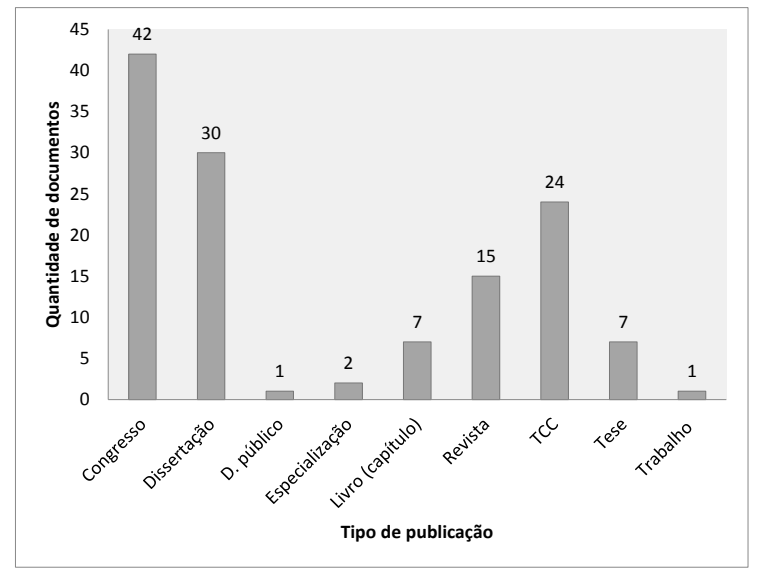

Fonte: Autores, 2019.
Gráfico 4 - Distribuição da quantidade de documentos por base de dados consultada

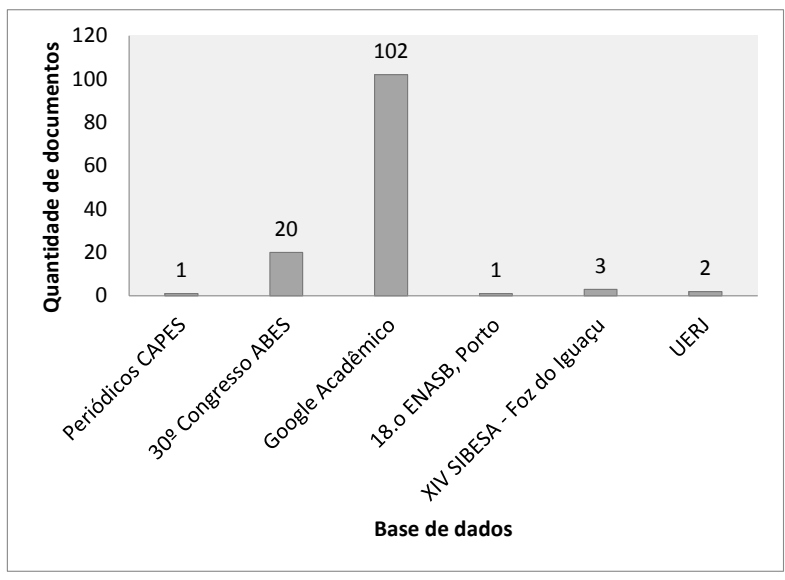

Fonte: Autores, 2019. 


\section{DISCUSSÃO}

Inicialmente observa-se (Gráfico 1) que a maioria dos trabalhos científicos publicados (72\%) adotam os dados do Atlas Esgotos apenas como citação e somente 5 trabalhos discorrem sobre o assunto e adotam os dados como fonte efetiva de consulta para desenvolvimento das suas pesquisas. Neste último caso, Soares et al. (2017) abordam o tema em um dos capítulos de uma importante publicação sobre a questão das dificuldades encontradas nos processos de licenciamento ambiental no Brasil para implantação de empreendimentos como fator colaborativo no desenvolvimento socioeconômico do país. Paulo et al. (2019) também abordaram os dados do Atlas Esgotos em capítulo de livro. Entretanto, neste caso, debateram a cerca da importância desse documento, como ferramenta de avaliação de sustentabilidade em sistemas de tratamento de esgotos descentralizados Ainda, os dados apresentados no Atlas Esgotos foram de grande importância para as pesquisas de ordem econômica estabelecidas nas publicações de Dutra e Smiderle (2018) e Dutra et al. (2018) e para a dissertação de mestrado de Sales (2018) que avaliou características de esgotamento sanitário em regiões rurais no Brasil, a partir dos dados publicados no Atlas.

Observa-se ainda em relação às publicações que de fato adotam o documento como fonte de pesquisa para desenvolvimento de seus trabalhos, que todas elas não se tratam de artigos científicos publicados em periódicos. Um detalhe importante a ser observado é a morosidade no processo de publicação de artigos em periódicos científicos no Brasil. Entre a submissão do artigo, a avaliação, a aceitação e a publicação de fato, em geral leva-se mais de 1 ano nos veículos de qualificação superior. A quantidade de documentos gerais é demasiadamente superior à quantidade de documentos do tipo artigo de periódico (Gráfico 3). Nota-se que no período analisado, dos 129 documentos que citam o Atlas Esgotos, somente 15 (12\%) são artigos de periódicos e 5\% oriundos de livros. Em quantidade bem significativa estão os trabalhos apresentados em Congressos (33\%), já que no recorte temporal ocorreram dois grandes eventos científicos da área de saneamento no Brasil. Já os trabalhos de conclusão de curso (graduação, especialização, mestrado e doutorado) representaram $50 \%$ dos trabalhos avaliados, isto é, a maioria. É importante avaliar esse destaque, considerando-se a importância do Atlas Esgotos para a formação de jovens profissionais do setor saneamento, que ainda encontram-se finalizando graduação ou cursos de especialização Latu Sensu. 
Essa tendência pode também ser observada na análise do Gráfico 2, onde percebe-se que a maioria dos documentos encontrados na pesquisa, foram publicados em 2018. É importante destacar que o Atlas Esgotos somente foi oficialmente lançado em setembro de 2017 e dessa forma já era de se esperar um número bastante reduzido de publicações no mesmo ano de lançamento do documento. Entende-se que muitas pesquisas que adotaram o Atlas Esgotos como fonte de consulta ainda podem não ter sido publicadas. Dos 3 documentos encontrados no ano de 2017, um deles é um capítulo de livro (Soares et al., 2017), outro é um trabalho de conclusão de curso de graduação (Santos, 2017), e outro um artigo de revista (Esteves, 2017). Além disso, a presente pesquisa foi finalizada em setembro de 2019 e, portanto, é possível que até a data de publicação, outros documentos já podem ter entrado nas bases de dados consultadas.

No gráfico 4, observa-se que $15 \%$ dos trabalhos avaliados encontram-se nos Anais do 30 CBESA ocorrido em 2019. Trata-se do evento científico de maior relevância para a área de Engenharia Sanitária no Brasil. Desses 20 trabalhos publicados nesse veículo, nenhum deles adota os dados do Atlas Esgotos para desenvolvimento da pesquisa; 70\% se apresenta no Objetivo 1 (Santos et al., 2019; Lima et al., 2019; Obraczka et al., 2019; Dantas et al., 2019; Caminada, 2019; do Amaral et al., 2019; Pereira et al., 2019; Brito et al., 2019; Brito et al., 2019; Volpato et al., 2019; Cavalcanti et al., 2019; Neves e Mol, 2019a; Silva et al., 2019; Pereira et al., 2019; Souza et al., 2019); e 30\% no Objetivo 2 (Santos et al., 2019; Silva Júnior et al., 2019a; Monteiro et al., 2019; Silva Júnior et al., 2019b; Motta Veiga, 2019; Pessoa et al., 2019).

É importante ainda salientar que outros temas de domínio foram abordados nos trabalhos consultados. Entretanto, como era de se esperar, de maneira maciça, os trabalhos discutem o tema saneamento (93\%). Os outros 7\% distribuem-se em temas como: saúde pública, direito, educação ambiental, energia, geografia e geoprocessamento (Prestes, 2018; Lannes et al., 2018; Maistro Spolidoro, 2018; Malzac e Silva, 2019; Santos AA et al., 2019; Parizotto, 2018; Cruz, 2018; Barra, 2018; Dias et al., 2018).

A partir da análise do Gráfico 4 observa-se claramente a grande abrangência do Google Scholar, uma vez que 102 das 129 publicações estudadas foram encontradas por meio desta plataforma, corroborando a conclusão de maiores abrangência e acessibilidade dessa plataforma (BROPHY e BAWDEN, 2005; MEIER e CONKLING, 2008; BUCHINGER et al., 2014; MEHO e YANG, 2007). 


\section{CONSIDERAÇÕES FINAIS}

O setor de ciência e tecnologia tem se apresentado cada vez mais em consonância com as ações de gestão e governança em diversos setores. Nesse sentido, a academia produz pesquisa de qualidade para a aplicação direta de resultados, dados e tecnologias no desenvolvimento socioeconômico do país. Ainda, o setor é responsável pela capacitação de recursos humanos, em todas as esferas da educação superior, como graduação, especialização, mestrado e doutorado. Nesse sentido, no âmbito da pesquisa apresentada, conclui-se que o documento Atlas Esgotos: Despoluição de Bacias Hidrográficas, objeto do presente estudo, vem se tornando uma ferramenta de grande relevância para o desenvolvimento da gestão de saneamento e recursos hídricos no Brasil, visto a sua grande inserção no ambiente técnico científico e acadêmico: 129 trabalhos técnico-científicos foram encontrados na pesquisa com a palavra chave "Atlas Esgotos" e suas variações, desde a publicação do documento até setembro de 2019; 20 trabalhos científicos, apresentados no primeiro grande evento de maior relevância no setor saneamento (30 $\mathrm{CBESA})$, após a publicação do documento, utilizam de alguma maneira os seus dados; 72 trabalhos foram publicados já no primeiro ano seguinte à publicação do Atlas; 50\% das publicações são referentes a trabalhos de conclusão de curso; 33\% dos trabalhos não somente citam os dados do Atlas Esgotos, mas sim discorrem sobre eles, utilizando-os no desenvolvimento de suas pesquisas ou minimamente comparando-os com outros dados apresentados por outras fontes.

Assim, diante do atual cenário de saneamento no território nacional, torna-se imprescindível o desenvolvimento de documentos públicos que retratem com fidelidade tal situação, de maneira a fomentar e propiciar a propagação de informações relativas ao saneamento básico e ao meio ambiente. Neste sentido, o Atlas Esgotos: Despoluição de Bacias Hidrográficas consolida-se como uma importante ferramenta informativa e como um poderoso instrumento estratégico de gestão, capaz de contribuir para o alcance da universalização dos serviços básicos de saneamento. 


\section{REFERÊNCIAS}

\section{Referências consultadas}

ANA - AGÊNCIA NACIONAL DE ÁGUAS. Atlas esgotos: despoluição de bacias hidrográficas. Brasília, 2017. Disponível em: $\quad$ http://arquivos.ana.gov.br/imprensa/publicacoes/ATLASeESGOTOSDespoluicaodeBaciasHidrograficasResumoExecutivo_livro.pdf $>$. Acesso em: 09 mar. 2020.

BATTISTELLA, P. E.; GRESSE VON WANGENHEIM, C.; FERNANDES, J. M. Como jogos educacionais são desenvolvidos? Uma revisão sistemática da literatura. In: XXII WORKSHOP SOBRE EDUCAÇÃO EM COMPUTAÇÃO, Brasília-DF. Anais. 2014. Disponível em: <http://repositorium.sdum.uminho.pt/bitstream/1822/33145/1/2014-WEI.pdf>. Acesso em: 09 mar. 2020.

BRASIL. Lei no 11.445 de 5 de janeiro de 2007. Estabelece as diretrizes nacionais para o saneamento básico e para a política federal de saneamento básico. Diário Oficial da União, Brasília, 11 jan. 2007.

BRASIL. Ministério do Desenvolvimento Regional. Secretaria Nacional de Saneamento - SNS. Sistema Nacional de Informações sobre Saneamento: 24ํDiagnóstico dos Serviços de Água e Esgotos - 2018. Brasília: SNS/MDR, 2019.

BROPHY, J.; BAWDEN, D. Is Google enough? Comparison of an internet search engine with academic library resources. Aslib Proceedings, v. 57, n. 6, p. 498-512, 2005. Disponível em: <https://pdfs.semanticscholar.org/f60e/c57c82296aebcab0cba8a8707670f94e60bf.pdf?_ga=2.227149725.1645039563.1 583782199-693597735.1581085212>. Acesso em: 09 mar. 2020.

BUCHINGER, D.; CAVALCANTI, G. A. S.; HOUNSELL, M. S. Mecanismos de busca acadêmica: uma análise quantitativa. Revista Brasileira de Computação Aplicada, v. 6, n. 1, p. 108-120, 2014. Disponível em: < http://seer.upf.br/index.php/rbca/article/view/3452/2548>. Acesso em: 09 mar. 2020.

COOK, D. J.; MULROW, C. D.; HAYNES, R. B. Systematic Reviews: Synthesis of Best Evidence for Clinical Decisions. Annals of Internal Medicine, v. 126, n. 5, p. 376-380, 1997. Disponível em: <https://annals.org/aim/articleabstract/710356/systematic-reviews-synthesis-best-evidence-clinical-decisions>. Acesso em: 09 mar. 2020.

COSTA, S. A. B. Auditoria e certificação das informações do sistema nacional de informações sobre saneamento (SNIS): um caminho necessário para a melhoria da qualidade da regulação e da gestão no setor saneamento. In: IX CONGRESSO BRASILEIRO DE REGULAÇÃO, Brasília-DF. Anais. 2015. Disponível em: <http://www.arsae.mg.gov.br/images/Img_Artigos/ABAR_2015_AuditoriaeCertificacaoSNIS.pdf>. Acesso em: 09 mar. 2020.

HELLER, L.; NASCIMENTO, N. O. Pesquisa e Desenvolvimento na Área de Saneamento no Brasil: necessidades e tendências. Engenharia Sanitária e Ambiental, Rio de Janeiro, v. 10, n. 1, p. 24-35, 2005. Disponível em: <http://www.scielo.br/pdf/esa/v10n1/v10n01a03.pdf>. Acesso em: 09 mar. 2020.

LISBOA, S. S.; HELLER, L.; SILVEIRA, R. B. Desafios do planejamento municipal de saneamento básico em municípios de pequeno porte: a percepção dos gestores. Engenharia Sanitária e Ambiental, Rio de Janeiro, v.18, n.4, p. 341-348, 2013. Disponível em: <http://www.scielo.br/pdf/esa/v18n4/1413-4152-esa-18-04-00341.pdf>. Acesso em: 09 mar. 2020.

MEHO, L. I.; YANG, K. Impact of data sources on citation counts and rankings of LIS Faculty: Web of Science vs. Scopus and Google Scholar. Journal of the American Society for Information Science and Technology, v. 58, n. 13, p. 2105 - 2125, 2007. Disponível em: <https://onlinelibrary.wiley.com/doi/pdf/10.1002/asi.20677>. Acesso em: 09 mar. 2020.

MEIER, J. J.; CONKLING, T. W. Google Scholar's coverage of the engineering literature: an empirical study. Journal of Academic Librarianship, v. 34, n. 3, p. 196-201, 2008. Disponível em: <https://www.sciencedirect.com/science/article/abs/pii/s0099133308000335 >. Acesso em: 09 mar. 2020.

SAMPAIO, R. F.; MANCINI, M. C. Estudos de revisão sistemática: um guia para síntese criteriosa da evidência científica. Revista Brasileira de Fisioterapia, São Carlos, v. 11, n. 1, p. 83-89, 2007. Disponível em: <http://www.scielo.br/pdf/rbfis/v11n1/12.pdf $>$. Acesso em: 09 mar. 2020. 
YÉVENES-SUBIATRE, A. Prospectiva y estrategia en el escenario contemporáneo. Latin American Journal of International Affairs, v. 2, n. 3, p. 90-106, 2010. Disponível em: <https://www.lajia.net/lajia/article/view/16/14>. Acesso em: 09 mar. 2020.

\section{Referências revisadas}

AGUIAR, R. A. MOREIRA, F. D.; MATTO, M. M.; DIAS, E. H. O. Diagnóstico das estações de tratamento de esgoto com reatores UASB no Brasil. In: 1 SEMINÁRIO NACIONAL SOBRE ESTAÇÕES SUSTENTÁVEIS DE TRATAMENTO DE ESGOTO, Curitiba-PR. Anais. 2018.

ALENCAR, C. T. G.; ZANETI, I. C. B. B. Resíduos sólidos e a microrregião de Ceres-GO: evolução histórica de uma realidade atual. In: IX SIMPÓSIO NACIONAL DE CIÊNCIA E MEIO AMBIENTE, Anápolis-GO. Anais. 2018.

ALMEIDA, I. R. Análise de vulnerabilidade à carência de esgotamento sanitário e sua gestão em municípios do Rio Grande do Sul. Dissertação - Universidade Federal do Rio Grande do Sul, 2019.

ALMEIDA, J. B. S. A. Educação ambiental e otimização da gestão de recursos hídricos em escolas rurais de ensino fundamental no município de Pacajus, CE. Monografia - Instituto Federal do Ceará, 2018.

AMARAL, A. F.; TAVARES, D. C. Potencialidade do reator biológico de leito móvel: revisão bibliográfica. In: IX CONGRESSO BRASILEIRO DE GESTÃO AMBIENTAL, São Bernardo do Campo/SP. Anais. 2018.

AMBROZEVICIUS, A. P. Diretrizes para implantação de tratamento de esgoto doméstico na Bacia Hidrográfica do Rio Paraíba do Sul. Monografia - Instituto Federal de Educação, Ciência e Tecnologia do Ceará, 2018.

ARAUJO, B. M.; SILVA JUNIOR, L. C. S. da; SANTOS A. S. P.; BILA, D. M. Comparativo econômico entre o custo estimado do reuso do efluente de ETE pra fins não potáveis e o valor da água potável para a bacia do rio paraíba do sul. In: III SIMPÓSIO DE RECURSOS HÍDRICOS DA BACIA DO PARAÍBA DO SUL, Juiz de Fora/MG. Anais. 2018 b.

ARAUJO, B. M.; SANTOS A. S. P.; LIMA, M. A. M.; RAMOS, L. T. A. Relação entre Capacidade de Reuso de Efluentes e a Demanda Hídrica para Irrigação no Rio de Janeiro. In: $18^{\circ}$ SILUBESA, Porto/PT. Anais. 2018a.

ARAÚJO, L. C.; GUIMARÃES, B. da S.; GALLINDO, A. de A. S.; FRANÇA, M. I. C. Avaliação da qualidade microbiológica da água da lagoa da Universidade Federal de Campina Grande - Paraíba. João Pessoa-PB: IMEA, v. 1, p. 205-220, 2019.

ARMANI, F. A. S.; FERREIRA, L. R. A.; OLIVEIRA, F. M. M. de; GOETZE, P. F. B.; SILVA, C. A. Qualidade das águas dos rios urbanos das cidades de Matinhos-PR e Pontal do Paraná-PR. Revista Técnico Científica do CREA-PR, v. 10, n.1, p. 11-10, 2018.

ÁVILA, Á. R. S. Melhoria da qualidade da água de lagos urbanos com uso de Wetland construído. Monografia Instituto Federal de Educação, Ciência e Tecnologia de Goiás, 2018.

BARRA, V. O. Site educacional para o ensino transdisciplinar das ciências ambientais. Mestrado profissional em Ciências Ambientais. Dissertação - Universidade Federal do Amazonas, 2018.

BONETTI, J. Análise da influência da taxa de infiltração nos custos de implantação de um sistema de esgotamento sanitário. Monografia - Universidade Federal de Santa Catarina, 2018.

BORBA, A. L. B.; FRANCO, P. L. P. Estações de tratamento de esgoto com reatores anaeróbios e filtros percoladores e as perspectivas para realização de nitrificação e para obtenção de padrões para reuso direto não potável em ambiente urbano e agrícola. In: 1ำ SEMINÁRIO NACIONAL SOBRE ESTAÇÕES SUSTENTÁVEIS DE TRATAMENTO DE ESGOTO, Curitiba-PR. Anais. 2018.

BRINGER, L. M.; REIS, J. A. T. dos; MENDONÇA, A. S. F. Wastewater treatment systems selection inside watersheds by using multiobjective analysis. Revista Brasileira de Recursos Hídricos, v. 23, n. 22, 2018.

BRITO, L. M. Cenário Comparativo das Áreas de Preservação Permanente da Bacia do Ribeirão José Pereira baseado no Novo Código Florestal e seus Possíveis Impactos na Condutividade Hidráulica do Solo. Monografia - Universidade Federal de Itajubá, 2018. 
BRITO, P. C.; KLIGERMAN, D. C.; OLIVEIRA, J. L. da M. Desempenho de um reator de leito móvel (MBBR) em escala de bancada com diferentes concentrações de carga orgânica. In: $30^{\circ}$ CONGRESSO BRASILEIRO DE ENGENHARIA SANITÁRIA E AMBIENTAL, Natal. Anais. 2019.

BUCCO, F. An evaluation of the environmental and energetic performance of a small-scale wastewater treatment plant with low waste production operated by solar energy. Dissertação - Universidade Federal do Paraná, 2018.

BUCCO, F.; AMARAL, K. J. do; NEUFFER, D.; MENZEL, U. Avaliação e adaptação do desempenho ambiental de uma estação de tratamento de efluentes compacta e energeticamente autárquica. In: $29^{\circ}$ CONGRESSO NACIONAL DE SANEAMENTO E MEIO AMBIENTE, São Paulo. Anais. 2018.

BURSZTEJN, S. A regulação do saneamento no Brasil e sua interface com a gestão dos recursos hídricos. 2018. 125p. Dissertação - Universidade Federal do Rio Grande do Sul, 2018.

CABRAL, D. M. L. Diagnóstico do abastecimento de água e esgotamento sanitário do município de Manaíra-PB. Monografia - Universidade Federal da Paraíba, 2018.

CALLISTO, M.; MORENO, P.; MACEDO, D. R. Biomonitoramento e pressões da urbanização: Uma abordagem integrada entre Ecologia e Geografia na bacia do rio das Velhas. Revista Espinhaço, v. 8, n. 1, p. 2-12, 2019.

CAMARA, L. R. A.; SILVA, D. D. S. da; SALES, L. L. N.; SILVA, D. W. S.; PINHEIRO, E. M. Qualidade de vida e percepção ambiental dos moradores de comunidades rurais de São Luís/MA. Revista Brasileira de Educação Ambiental, v. 14, n. 1, p. 263-274, 2019.

CAMINADA, S. M. L.; PAGANINI, W. da S.; BOCCHIGLIERI, M. M. Avaliação da presença de fármacos em biossólido compostado proveniente de ETE e suas implicações na Saúde Pública. In: 30 CONGRESSO BRASILEIRO DE ENGENHARIA SANITÁRIA E AMBIENTAL, Natal. Anais. 2019.

CARLI, B. P. Zooplâncton como indicador da qualidade ambiental em reservatórios do estado de São Paulo. Tese (Doutorado) - Universidade Estadual Paulista, 2019.

CARVALHO JÚNIOR, R. P. Avaliação da eficiência do sistema de lagoas de alta taxa como pós-tratamento dos reatores UASB para garantia da qualidade do efluente final - ETE Paranoá/DF. Monografia - Universidade de Brasília, 2018.

CAVALCANTI, A. L. A.; KOPP, K.; HORA, K. E. R. Doenças relacionadas ao saneamento ambiental inadequado na região metropolitana de Goiânia (GO) entre 2008 e 2018. In: 30 CONGRESSO BRASILEIRO DE ENGENHARIA SANITÁRIA E AMBIENTAL, Natal. Anais. 2019.

CERQUEIRA, R. J. A. Os desafios na execução do Programa de Aceleração do Crescimento (PAC): o caso do esgotamento sanitário. Dissertação - Fundação Oswaldo Cruz, 2019.

CHERNICHARO, C. A. L.; RIBEIRO, T. B.; GARCIA, G. B.; LERMONTOV, A.; PLATZER, C. J.; POSSETTI, G. R. C.; LEITES, M. A. L.; ROSSETO, R. Panorama do tratamento de esgoto sanitário nas regiões Sul, Sudeste e Centro-Oeste do Brasil: tecnologias mais empregadas. Revista DAE, v. 66, n. 213, p. 5-19, 2018.

CORRÊA, J. M. M. Avaliação da presença de microcontaminantes de preocupação emergente, por GC-MS, e de elementos inorgânicos, por TXRF, nas águas superficiais da Bacia do Rio Paraopeba/MG. Dissertação Universidade Federal de Ouro Preto, 2018.

CORRÊA, R. C. L.; ARAÚJO, F.de C. D.; SILVA, S. J. da; MAJEROWICZ, N.; DIAS, A. Qualidade da água de poços em unidades de produção familiar nos municípios de Itaguaí e Seropédica (RJ). João Pessoa-PB: IMEA, v. 1, p. 93-110, 2019.

CRUZ, B. N. Principais complicações parasitárias causadas por cryptosporidium parvum, isospora belli e microsporídios em pacientes imunocomprometidos. Monografia - Centro Universitário de Brasília, 2018. 
DANTAS, M. S.; OLIVEIRA, J. C.; BARROSO, G. R.; SOARES, A. L. C.; OlIVEIRA, S. M. A. C. Avaliação comparativa de estações de tratamento de esgotos por UASB e filtro biológico percolador entre duas bacias hidrográficas de Minas Gerais. In: 30 CBESA, Natal. Anais. 2019.

DE SOUSA CARRASCO, R. Proposição de cenário de reuso de águas amarelas em um assentamento rural de Santa Catarina. Monografia - Universidade Federal de Santa Catarina, 2018.

DIAS, I. C. Indicadores de sustentabilidade de bacia hidrográfica e hidroquímica de poços no estado do Maranhão: subsídios ao gerenciamento e conservação dos recursos hídricos. Tese (Doutorado) - Universidade Federal do Maranhão, 2018.

DIAS, I. C. L.; FRANÇA, V. L.; BEZERRA, D. S.; RABÊLO, J. M. M.; CASTRO, A. C. L. Spatial distribution of river basin sustainability indicators in transition region of northeastern Brazil. Applied Ecology and Environmental Research, v. 16, n. 4, p. 3729-3754, 2018.

DIAS, N. A. Déficit de acesso à coleta de esgoto sanitário no Espírito Santo. Dissertação - Universidade Federal do Espírito Santo, 2018.

DO AMARAL, K. G. C. Avaliação da sustentabilidade no gerenciamento do lodo e biogás, em estação de tratamento de esgoto, utilizando a técnica de ACV. Tese (Doutorado) - Universidade Federal do Paraná, 2018.

DO AMARAL, K. G. C.; AISSE, M. M.; POSSETTI, G. R. C. Análise do custo de ciclo de vida inerente ao tratamento e destinação final do lodo biológico e biogás, provenientes de ETE que emprega reator UASB. In: XIV - SIBESA, Foz do Iguaçu. Anais. 2018.

DO AMARAL, K. G. C.; AISSE, M. M.; POSSETTI, G. R. C.Avaliação da sustentabilidade do lodo e biogás em estação de tratamento de esgoto. In: $30^{\circ}$ CONGRESSO BRASILEIRO DE ENGENHARIA SANITÁRIA E AMBIENTAL, Natal. Anais. 2019.

DUARTE, S. C. Ambiente regulatório do setor de saneamento no Brasil: Limites e possibilidades de atuação da Agência Nacional de Águas junto aos entes Federados Subnacionais. Monografia - Escola Nacional de Administração Pública, 2019.

DUTRA, JOISA; FREITAS, R. M. S.; SMIDERLE, J. J.; DIAS, S. A. B.; SOUZA, R. M.; ZIDDE, C. Medindo Saneamento Potencialidades e limitações dos bancos de dados brasileiros. Rio de Janeiro: FGV, 2018.

DUTRA, J.; SMIDERLE, J. J. Entendendo o desafio do saneamento no Brasil. Conjuntura Econômica, Rio de Janeiro, p. 60-62, 2018.

ESTEVES, C. J. O. A Aplicação dos Instrumentos da Política Nacional e Estadual de Recursos Hídricos no Paraná: sistematização de informações e abordagem panorâmica. Caderno IPARDES, v. 7, n.1, p. 20-61, 2017.

FARIA, E.; GIRARD, P.; NARDES, C. S.; MORESCHI, A.; CHRISTO, S. W.; FERREIRA JUNIOR, A. L.; COSTA, M. F. Microplastics pollution in the South American Pantanal. PeerJ Preprints, v. 7, n. 27754, 2019.

FERREIRA, D. de M. Avaliação de risco no reuso de esgotos domésticos utilizados na agricultura. Tese (Doutorado) - Universidade Federal do Rio Grande do Norte, 2019.

FIGUEIREDO, I. C. S. Tratamento de esgoto na zona rural: diagnóstico participativo e aplicação de tecnologias alternativas. Tese (Doutorado) - Universidade Estadual de Campinas, 2019.

FRANCI, T. K. Análise das condições para desenvolvimento do mercado de estruvita recuperada a partir de águas residuárias no Brasil. Dissertação - Universidade Federal do Espírito Santo, 2018.

FREIRE, A. F. Esgotamento sanitário na cidade de Araguaína (TO): uma correlação entre as principais doenças e a questão da vulnerabilidade. Dissertação - Universidade Federal do Tocantins, 2019.

GARCIA, D. P. Development of a classification instrument to allocate federal funding available for sanitation improvement in the cities under fifty thousand people in Ceará, Brazil. Tese (Doutorado) - University of Twente, Países Baixos, 2018. 
GUINZANI, G. V.; SAMPAIO, L. A. R.; SÁ, L. S. Estação de tratamento de água subterrânea para abastecimento do condomínio solar de Maricá I e proposta para utilização de águas cinzas. Monografia - Universidade Federal Fluminense, 2018.

ISSA, C. G. Testes ecotoxicológicos para avaliação do potencial impacto ambiental em corpos receptores por efluente de Estação de Tratamento de Esgoto. Dissertação - Universidade de São Carlos, 2019.

KATO, M. T.; LAPOLLI, F. R.; SOUSA, J. T.; NOLASCO, M. A.; GONÇALVES, R. F; LEITE, V. D. Oportunidades e desafios na implementação de estações de tratamento de esgoto descentralizadas. Fortaleza: Imprece, p. 23-47, 2019.

LANNES, L. R. O.; FONTOURA, G. A. T.; LERMONTOV, A.; PEREIRA, R. A. S. Análise comparativa da geração teórica e real de biogás de uma estação de tratamento de esgotos com sistema anaeróbio do tipo UASB. In: XIV - SIBESA, Foz do Iguaçu. Anais. 2018.

LEAL, J. da S. G. Wetland construído para tratamento de efluente secundário de condomínio empresarial. Dissertação - Universidade Federal de Uberlândia, Minas Gerais, 2019.

LIMA, B. A. de. Degradação da Hidroclorotiazida por processo UV $/ \mathrm{H}_{2} \mathrm{O}_{2}$. Monografia - Universidade Federal do Rio Grande do Norte, 2018.

LIMA, I. M. Utilização do modelo matemático QUAL-UFMG para avaliar a capacidade de autodepuração do rio Paraíba do Sul. Monografia - Universidade Federal do Rio de Janeiro, 2019.

LIMA, M. A. M. Avaliação do potencial de reuso de efluente de Estação de Tratamento de Esgotos para irrigação nas Regiões Hidrográficas Brasileiras. Monografia - Universidade do Estado do Rio de Janeiro, 2019.

LIMA, R.; LEAL, P. B.; LOPES, L. N.; BRANCACCIO, G. R.; RODRIGUES, R. C. L. S. Análise dos parâmetros físico-químicos e microbiológicos e a influência do uso e ocupação do solo dos rios João Mendes e Mombuca. In: $30^{\circ}$ CONGRESSO BRASILEIRO DE ENGENHARIA SANITÁRIA E AMBIENTAL, Natal. Anais. 2019.

LOPES, L. S. Avaliação do potencial de recuperação energética de lodo e biogás provenientes de reatores UASB tratando esgoto doméstico no estado do Paraná. Dissertação - Universidade Federal de Viçosa, 2018.

MACHADO, P. B. Conflitos pelo uso da água: papel mediador do Comitê da Bacia Hidrográfica do Lago de PalmasTO. Dissertação - Universidade Federal do Tocantins, 2019.

MAIA, F. Q. Aporte e avaliação de risco de atenolol, carbamazepina e sulfametoxazol no lago Paranoá. Monografia - Universidade de Brasília, 2018.

MAISTRO SPOLIDORIO, P. C. As contribuições do Direito Francês à gestão ecossistêmica das águas na zona costeira brasileira: o uso do plano de bacia hidrográfica como instrumento jurídico de integração. Dissertação - Universidade de Brasília, 2018.

MALZAC, M. E.; SILVA, T. C. da. Delimitações da zona costeira Sul do estado da Paraíba, Brazil. Revista de Gestão Costeira Integrada, v. 19, n. 2, p. 123-141, 2019.

MEDEIROS, V. S. Abordagem ecotoxicológica para avaliação de reatores anaeróbios com meio suporte e com microrganismos eficientes. Dissertação - Universidade Federal de Viçosa, 2019.

MEDEIROS, V. S.; SALEH, B. B.; AGUIAR, A. C. R.; FURQUIM FILHO, C. S.; ALVES, W. S.; MIRANDA, R. A.; AQUINO, D. S. Verificação da conformidade da qualidade do efluente da estação de tratamento de esgotos de Rio Verde/GO. Revista Gestão e Sustentabilidade Ambiental, v. 8, n.1, p. 814-831, 2019.

MELCHIOR, C. P.; LOPES, M. S.; OLIVEIRA, E. C. M.; AMBROSIO, J. K.; BRENTAN, B. M.; FAIS, L. M. C. F.; RIBEIRO, L. C. L. J. Estudo das Equações do Cálculo do Coeficiente de Dispersão usando Análise Correlacional. In: XXVI CONGRESSO DE INICIAÇÃO CIENTÍFICA DA UNICAMP, Campinas. Anais. 2018.

MIRANDA GONÇALVES, R.; DOMINGOS, I. M. N. População ribeirinha no Amazonas e a desigualdade no acesso à saúde. Revista de Estudos Constitucionais, Hermenêutica e Teoria do Direito, v. 11, n.1, p. 99-108, 2019. 
MONTEIRO, M. M.; BARÉA, P. B.; CUBAS, S. A.; AISSE, M. M. Filtro biológico percolador como pós-tratamento de efluentes anaeróbios: uma reflexão. In: $30^{\circ}$ CONGRESSO BRASILEIRO DE ENGENHARIA SANITÁRIA E AMBIENTAL, Natal. Anais. 2019.

MORETTO, M. R. S. D. Relação entre saúde pública e saneamento na bacia hidrográfica do Ribeirão Marinheiro, inserido na unidade de gerenciamento de recursos hídricos - 15, estado de São Paulo. Dissertação - Universidade Estadual Paulista, 2018.

MOTTA VEIGA, M. Universalização dos serviços públicos de saneamento básico no brasil: Fake News? In: $30^{\circ}$ CONGRESSO BRASILEIRO DE ENGENHARIA SANITÁRIA E AMBIENTAL, Natal. Anais. 2019.

NASCIMENTO, D. de B. do; GONÇALVES, R. da S. Coleta e tratamento de esgoto para pequenas comunidades-estudo de caso em áreas rurais. Monografia - Universidade do Sul de Santa Catarina, 2019.

NEVES, A. C.; MOL, M. P. G. Modelo teórico da avaliação de risco ambiental de fármacos consumidos em Belo Horizonte/MG. In: 30 CONGRESSO BRASILEIRO DE ENGENHARIA SANITÁRIA E AMBIENTAL, Natal. Anais. 2019a.

NEVES, A. C.; MOL, M. P. G. Theoretical environmental risk assessment of ten used pharmaceuticals in Belo Horizonte, Brazil. Environmental Monitoring and Assessment, v. 2019, p. 191-275, 2019b.

NEVES, P. N. P.; RIBEIRO, T. B.; CHERNICHARO, C. A. L. Lodo granular anaeróbio: uma possibilidade para inocular reatores UASB tratando esgoto sanitário? In: 1ำ SEMINÁRIO NACIONAL SOBRE ESTAÇÕES SUSTENTÁVEIS DE TRATAMENTO DE ESGOTO, Curitiba-PR. Anais. 2018.

OBRACZKA, M.; MURICY, B. C.; OHNUMA JÚNIOR, A. A.; MARQUES, C. F.; PINTO, S. O. M. Aperfeiçoamento da gestão do sistema de esgotamento sanitário com base em parâmetros e indicadores de saneamento. In: $30^{\circ}$ CONGRESSO BRASILEIRO DE ENGENHARIA SANITÁRIA E AMBIENTAL, Natal. Anais. 2019.

OLIVEIRA, B. V.S. de. Avaliação da eficiência energética em estações de tratamento de esgotos sanitários: estudo de caso no município de Jaraguá do Sul. Monografia - Universidade Federal de Santa Catarina, 2018.

OLIVEIRA, G. F. A.; SILVA, J. B.; MONTEIRO, G. F.; ARAÚJO, R. J. S.; SANTOS, M. B. H. Avaliação da qualidade da água destinada à irrigação e dessedentação animal. João Pessoa-PB: IMEA, v. 1, p. 188-205, 2019.

OLIVEIRA, J. C. Utilização de um biodigestor para tratamento de esgoto e geração de energia: um estudo de caso na comunidade de Portelinha, RJ. Monografia - Universidade Federal do Rio de Janeiro, 2018.

PALASIO, R. G. S. Padrão de distribuição da diversidade genética molecular e espacial de Biomphalaria e sua relação com a ocorrência da esquistossomose, região do médio Paranapanema, Estado de São Paulo. Tese (Doutorado) - Universidade de São Paulo, 2019.

PARIZOTTO, D. Planejamento estratégico: uma proposta de implantação para uma empresa do ramo elétrico. Monografia - Universidade Federal da Fronteira Sul, 2018.

PAULO, P. L.; KIPERSTOK, A.; SOUZA, H. H. S.; DE MORAIS, J. C., OLIVEIRA, L. V.; QUEIROZ, L. M.; NOLASCO, M. A.; MAGRI, M. E.; LOPES, T. S. Ferramentas de avaliação de sustentabilidade em sistemas de tratamento de esgotos descentralizados. Fortaleza: Imprece, p. 746-812. 2019.

PEIXOTO, A. de S.; SALLES, M. J.; MARTIN, L. E.; GRIMBERG, P. Ecotecnologia de Wetlands construídos: a eficiência das macrófitas emergentes no tratamento de efluentes em experiências brasileiras. In: $29^{\circ}$ CONGRESSO NACIONAL DE SANEAMENTO E MEIO AMBIENTE, São Paulo. Anais. 2018.

PEREIRA, A. C.; MARAGON, B. B.; SANTOS, T. C. C.; REZENDE, A. A. P. Definição de critérios para escolha de alternativas de sistemas individuais de esgotamento sanitário. In: $30^{\circ}$ CONGRESSO BRASILEIRO DE ENGENHARIA SANITÁRIA E AMBIENTAL, Natal. Anais. 2019.

PEREIRA, F. C.; MARTINS, R. T. A. A.; GONÇALVES, M. M. Projeto de captação de água subterrânea para expansão do sistema de abastecimento de água do condomínio residencial total life. In: $30^{\circ}$ CONGRESSO BRASILEIRO DE ENGENHARIA SANITÁRIA E AMBIENTAL, Natal. Anais. 2019. 
PERTUSSATTI, C. A. Revisão do plano nacional de resíduos sólidos-evolução da política nacional de resíduos sólidos. Monografia - Instituto Federal de Educação, Ciência e Tecnologia do Ceará, 2018.

PESSOA, L. M.; OLIVEIRA, M. D.; DE PAULA, F. S. Avaliação do lançamento de esgoto sanitário na Bacia Hidrográfica do Rio São Francisco em Minas Gerais. In: II SIMPÓSIO DA BACIA HIDROGRÁFICA DO RIO SÃo FRANCISCO, Aracajú. Anais. 2018.

PESSOA, L. M.; DE PAULA, F. S.; MARQUES, M. L. G. V. Estimativa de investimentos em tratamento de esgoto por bacia hidrográfica de Minas Gerais. In: $30^{\circ}$ CONGRESSO BRASILEIRO DE ENGENHARIA SANITÁRIA E AMBIENTAL, Natal. Anais. 2019.

PIGOZZO, C. Caracterização do efluente hospitalar produzido em dois hospitais da cidade de Porto Alegre/RS. Monografia - Universidade Federal do Rio Grande do Sul, 2018.

PIZUTTI, J. T. Pós-tratamento de efluente de reator UASB por eletrocoagulação e adsorção para reuso. Dissertação - Universidade de Passo Fundo, 2018.

PONTES FILHO, J. D. de A. Da recarga não gerenciada à recarga gerenciada: estratégia para aquífero aluvial no semiárido brasileiro. Dissertação - Universidade Federal de Campina Grande, 2018.

PRESTES, W. de L. Proposta de monitoramento para um biodigestor residencial e criação de dados sobre produção de biometano e geração de créditos de carbono. Monografia - Centro Universitário Autônomo do Brasil, 2018.

RAMOS, M. C. A. F.; SANTOS, J. M. B.; SILVA, W. R.; CHERNICHARO, C. A. L.; GALÉRY, R.; BRANDT, E. M. F. Adsorventes desenvolvidos a partir de resíduos da mineração de ferro e da siderurgia para o beneficiamento de biogás. In: $1^{\circ}$ SEMINÁRIO NACIONAL SOBRE ESTAÇÕES SUSTENTÁVEIS DE TRATAMENTO DE ESGOTO, Curitiba. Anais. 2018.

REIS, G. B.; ABREU, V. O.; FRAGA, M. S.; TOZI, T.; SILVA, D. D. Proposta de enquadramento de corpos hídricos em classes de uso na bacia hidrográfica do rio Santo Antônio (MG). Nativa, v. 7, n.2, p. 189-196, 2019.

RIBEIRO, A. A. S.; DUARTE, A. D.; BEZERRA, S. T. M.; COELHO, I. C. L.; GONÇALVES, E. A. P. Índice de salubridade ambiental em áreas urbanas: um estudo em Caruaru-PE. In: 29 CONGRESSO NACIONAL DE SANEAMENTO E MEIO AMBIENTE, São Paulo. Anais. 2018.

ROSA JÚNIOR, L. A.; SOUZA, C. L.; MACHADO, L. M. Caracterização de hidrogramas diários de vazão de esgoto doméstico em condomínio de Belo Horizonte. In: XIV-SIBESA, Foz do Iguaçu. Anais. 2018.

ROSÁRIO, L. N.; FERNANDES, J. G. Estudo de autodepuração do rio Paraíba do Sul após lançamento do efluente de uma ETE. In: XV SIMPÓSIO DE EXCELÊNCIA EM GESTÃO E TECNOLOGIA, Resende. Anais. 2018.

SALES, B. M. Caracterização dos Determinantes da Exclusão Sanitária nos Domicílios Rurais Brasileiros. Dissertação - Universidade Federal de Minas Gerais, 2018.

SANTOS, A. de A.; SOARES, C. M.; SANTOS, E. C. S.; TANI, K. A.; FERREIRA, P. J. Estudo de caso - Desastre ambiental da barragem de fundão na cidade de Mariana-MG causado pela Samarco: Análise do município de Governador Valadares-MG. Trabalho elaborado no âmbito da disciplina, 2019.

SANTOS, A. S. P.; LIMA, M. A. M.; RAMOS, L. T. A.; SOARES, S. R. A.; PEREIRA, C. B. Avaliação do potencial de reuso de efluente de ETE para o setor agrícola na Bacia Hidrográfica do Rio Grande. In: $30^{\circ}$ CONGRESSO BRASILEIRO DE ENGENHARIA SANITÁRIA E AMBIENTAL, Natal. Anais. 2019.

SANTOS, A. S. P.; LIMA, M. A. M.; RAMOS, L. T. A.; SOARES, S. R. A.; PEREIRA, C. B.; MELO, M. C. Capacidade de Reuso de Efluentes de Estações de Tratamento de Esgoto na Bacia Hidrográfica do Paraíba do Sul. Semioses, v. 12, n.3, p. 16, 2018a.

SANTOS, A. S. P.; LIMA, M. A. M.; RAMOS, L. T. A.; SOARES, S. R. A.; PEREIRA, C. B.; MELO, M. C. Estimativa da Capacidade disponível de reuso de efluentes na bacia hidrográfica do paraíba do sul. In: III SIMPÓSIO DE RECURSOS HÍDRICOS DA BACIA DO PARAÍBA DO SUL, Juiz de Fora. Anais. 2018b. 
SANTOS, F. N. B.; MOTA JÚNIOR, C. R.; JÚDICE, M. A. M.; SALIBA, P. D. Análise dos custos operacionais de 44 estações de tratamento de esgoto. In: $30^{\circ}$ CONGRESSO BRASILEIRO DE ENGENHARIA SANITÁRIA E AMBIENTAL, Natal. Anais. 2019.

SANTOS, S. H. de J. Avaliação de um Wetland: sistema alagado construído para tratamento de esgoto. Monografia - Instituto Federal de Sergipe, 2017.

SILVA JÚNIOR, L. C. S.; AZEVEDO, J. P. S.; SANTOS, A. S. P.; ANDRADE, V. S.; MELO, M. C. Diagnóstico do esgotamento sanitário e qualidade de água da bacia hidrográfica do rio Piabanha. In: $30^{\circ}$ CONGRESSO BRASILEIRO DE ENGENHARIA SANITÁRIA E AMBIENTAL, Natal. Anais. 2019a.

SILVA JÚNIOR, L. C. S; ARAUJO, B. M.; SANTOS, A. S. P.; OBRACZKA, M.; BOTTREL, S. E. C. Panorama do reuso de efluentes nas estações de tratamento de esgotos da região sudeste brasileira. In: $30^{\circ}$ CONGRESSO BRASILEIRO DE ENGENHARIA SANITÁRIA E AMBIENTAL, Natal. Anais. 2019b.

SILVA JÚNIOR, L. C. S.; TRINDADE, A. B. Análise da produção de energia elétrica a partir do biogás gerado em reatores anaeróbios de estações de tratamento de esgoto brasileiras. In: 1ํ SEMINÁRIO NACIONAL SOBRE ESTAÇÕES SUSTENTÁVEIS DE TRATAMENTO DE ESGOTO, Curitiba. Anais. 2018.

SILVA, A. P. V. da. Os efeitos do ICMS Ecológico para as políticas ambientais dos municípios fluminenses. Tese (Doutorado) - Universidade Federal do Rio de Janeiro, 2018.

SILVA, D. L. da. Avaliação do Plano Municipal de Saneamento Básico de Maricá com vistas à sua revisão. Monografia - Universidade Federal do Rio de Janeiro, 2019.

SILVA, J. I. A. O.; LEITE, J. R. M. Public Management as a Risk Causator in Brazilian metropolis: The case of Florianópolis. In: I-REC2019 DISRUPTING THE STATUS QUO: RECONSTRUCTION, RECOVERY AND RESISTING DISASTER RISK CREATION. Flórida. Anais. 2019.

SILVA, J. S. Remoção de matéria orgânica em tanque séptico com biomassa aderida. Dissertação - Universidade Tecnológica Federal do Paraná, 2018.

SILVA, L. P. A.; SANTOS, H. R.; BARROS FILHO, M. A. C.; FREITAS, F. R. S. Pós-tratamento de efluente sanitário com membrana de ultrafiltração visando reúso urbano não potável. In: $30^{\circ}$ CONGRESSO BRASILEIRO DE ENGENHARIA SANITÁRIA E AMBIENTAL, Natal. Anais. 2019.

SILVA, N. S. Efeito da urbanização nas características geoquímicas da laguna de Carapebus - Serra/ES. Dissertação - Universidade Federal do Espírito Santo, 2018.

SILVA, R. M. Q. Extração por partição em baixa temperatura para determinação simultânea de microcontaminantes de preocupação emergente em esgoto bruto total por CG/EM. Dissertação - Universidade Federal de Ouro Preto, 2018.

SILVEIRA, A. C. B. Estudo de Caso da Implantação do Sistema Separador Absoluto de Esgoto em Sub-bacias do Sistema Marangá, Município do Rio de Janeiro, sob uma Perspectiva de Saúde Ambiental. Dissertação - Pontifícia Universidade Católica do Rio de Janeiro, 2018.

SILVEIRA, S. F. Avaliação da gestão de resíduos sólidos urbanos no município de Itabira (MG): uma ênfase na coleta seletiva. Dissertação - Universidade Federal de Ouro Preto, 2018.

SIQUEIRA JUNIOR, C. R. de. Sistema alternativo de tratamento de esgoto para residências unifamiliares em comunidade carente no Município de Maceió/AL. Monografia - Centro Universitário CESMAC, 2018.

SOARES, S. R. A.; FONTENELLE, T. H.; ANDRADE, L. A. Z. de; SILVA, M. A.; PEREIRA, C. B. Subsídios do planejamento de recursos hídricos para o licenciamento ambiental. Rio de Janeiro: IPEA, p. 107-118, 2017.

SOARES, T. L. Abundância e distribuição de elementos metálicos em águas integrantes da Unidade de Planejamento e Gestão de Recursos Hídricos Caratinga, sub-bacia do rio Doce. Dissertação - Universidade Federal de Ouro Preto, 2019. 
SOUZA, H. R. de. Saneamento rural sustentável: sistemas alternativos para a região dos Sertões de Crateús. Monografia - Universidade Federal do Ceará, 2019.

SOUZA, M. C. de. Avaliação da prática do reúso com esgoto tratado em lagoas de estabilização no semiárido do Rio Grande do Norte. Dissertação - Universidade Federal do Rio Grande do Norte, 2018.

SOUZA, T. C.; OLIVEIRA, J. L. M.; SAGGIORO, E. M.Remoção de nutrientes e $17 \alpha$ Etinilestradiol (EE2) em esgoto por reator em batelada sequencial anaeróbio-aeróbio-anóxico. In: $30^{\circ}$ CONGRESSO BRASILEIRO DE ENGENHARIA SANITÁRIA E AMBIENTAL, Natal. Anais. 2019.

TEIXEIRA, J. I. R. A Problemática do Investimento Estrangeiro nos Direitos Humanos 0 direito à água e ao saneamento básico. Dissertação - Universidade Portucalense, 2018.

TEUBNER JÚNIOR, F. J.; LIMA, A. T. M.; BARROSO, G. F. Emission rates of nitrogen and phosphorus in a tropical coastal river basin: a strategic management approach. Environmental Monitoring and Assessment, v.190, n. 747. 2018.

VIANA, A. M. Bioindicadores na avaliação da capacidade de autodepuração em um período de seca do Rio Grande - Diamantina - MG. Dissertação - Universidade Federal dos Vales do Jequitinhonha e Mucuri, 2018.

VIANA, M. O.; PEREIRA JÚNIOR, P. G. P.; COSTA, T. S.; SANTOS, M. B. H. Análises físico-químicas de água consumida em um bebedouro da Universidade Federal da Paraíba, Campus II. João Pessoa-PB: IMEA, v. 1, p. 151-170, 2019.

VIEIRA, J. H. WasteWater Treatment in the recharge and discharge areas of the Guarani Aquifer. In: XX CONGRESSO BRASILEIRO DE ÁGUAS SUBTERRÂNEAS. Anais. 2018.

VOLPATO, E. L.; RODRIGUES, F. N.; FIA, R.; FIA, F. R. L. Determinação da qualidade da água e análise de correlação entre as variáveis de qualidade do Ribeirão Vermelho em Lavras- MG. In: 30 CONGRESSO BRASILEIRO DE ENGENHARIA SANITÁRIA E AMBIENTAL, Natal. Anais. 2019

ZANETONI FILHO, J. A. Cultivo de landoltia punctata na remoção de desreguladores endócrinos e no polimento de esgoto sanitário em lagoa com recirculação. Dissertação - Universidade Estadual Paulista, 2019.

\section{(cc) EY}

Este trabalho está licenciado com uma Licença Creative Commons - Atribuição 4.0 Internacional. 